\title{
Camera-based sample-position detection and control for microgravity electrostatic levitation
}

\author{
D. Bräuer ${ }^{1, \text { a) }}$ and C. Neumann ${ }^{1}$ \\ Institut für Materialphysik im Weltraum, Deutsches Zentrum für Luft- und Raumfahrt (DLR), 51170 Köln, \\ Germany
}

(Dated: 26 March 2020)

\begin{abstract}
This paper presents a method for high-speed sample detection and position control in an electrostatic levitator. The algorithm uses images acquired from two CCD (charge coupled device) cameras and allows robust and reliable detection of the sample position under various process conditions. The results show improvements over PSD (position sensitive detector) systems especially under harsh environments and during autonomous operation under microgravity conditions. The position of samples with a radius from $0.6 \mathrm{~mm}$ to $1.1 \mathrm{~mm}$ is detected in three dimensions with an accuracy of $\pm 40 \mu \mathrm{m}$ inside a $7 \mathrm{~mm} \times 7 \mathrm{~mm} \times 7 \mathrm{~mm}$ levitation area. The two orthogonally arranged cameras, recording images at a resolution of $260 \mathrm{px} \times 260 \mathrm{px}$, are used to calculate the position every $5 \mathrm{~ms}$. The control model and the corresponding position controller for the three axes are presented as well. The system was successfully tested in laboratory and under microgravity conditions at the drop tower, during parabolic flights, and on the MAPHEUS sounding rocket.
\end{abstract}

\section{INTRODUCTION}

Electrostatic levitation technique allows for investigation of thermophysical properties of melts at high temperatures. Material properties such as viscosity or density can be determined with high precision over a wide range of temperatures, including a deep undercooling ${ }^{1-11}$. Due to the lack of potential minima in electrostatic fields as stated by the Earnshaw Theorem ${ }^{12}$ the sample position can never be autostabilising. A feedback controller is mandatory to sustain stable levitation and the determination of position is time critical for this process. Therefore, a fast and precise measurement of the three dimensional sample position has to be implemented.

This work contributes to a project of electrostatic levitation in microgravity environment which eliminates possible gravitational influence and gains benchmark results for groundbased experiments. In particular an instrument to perform on the sounding rocket MAPHEUS is being designed ${ }^{13}$. A key issue for this task is to set up a compact and robust position detection and control system offering a high grade of automation and reliability.

While electrostatic levitation on ground was used for several years ${ }^{14}$, electrostatic levitation under microgravity conditions is still work in progress. In 2016 the JAXA Electrostatic Levitation Furnace (ELF) ${ }^{15,16}$ was installed in the Kibō module at the International Space Station and a first functional checkout was performed $^{17}$.

The facility described here uses a different setup with only four controllable electrodes and high resolution CCD cameras to control the sample position.

\section{SAMPLE DETECTION}

To identify the sample position, either positions sensitive detectors (PSDs) or imaging devices can be used. A PSD is

\footnotetext{
a)Electronic mail: dirk.braeuer@dlr.de
}

an area-photo-diode where incident light generates a current in the illuminated sector. By measuring these currents the position of a light point can be calculated ${ }^{1,18}$. The amplified output signal of the sensor is an analogue signal representing the centre of the incident light. Therefore, PSDs can be used to measure the position of a sample. To determine $3 \mathrm{~d}$ position information two sensors are employed and arranged orthogonally ${ }^{1,19}$.

The analogue signal allows fast and continuous measurement of sample positions. These PSD sensors offer good performance in laboratory environments. For environmental conditions on microgravity platforms they turned out to be not reliable due to thermal drift, increased g-levels and vibrations. During the $25^{\text {th }}$ DLR Parabolic flight campaign a prototype of the microgravity ESL facility using PSD sensors was tested. In laboratory environment the devices can be adjusted manually for each experiment. Under microgravity on parabolic flights or on sounding rockets manual adjustment is not possible.

To overcome those problems the PSDs were replaced by two CCD (charge coupled device) cameras. First experiences with CCD-based measurement of sample position are given in Ref. 14. The authors used a $50 \mathrm{px} \times 50 \mathrm{px}$ CCD to detect the sample in a range of $10 \mathrm{~mm} \times 10 \mathrm{~mm}$. A threshold black and white image is derived from the acquired image in order to detect the samples position. It turned out that the resulting optical resolution of $0.2 \mathrm{~mm}$ and the performance of the image analysis were unsatisfying to control levitation ${ }^{20}$.

Nowadays, the performance of CCD camera systems and image analysis allows to obtain the sample position at higher frame rates and higher resolution. In addition, the camera system makes it easier to observe the behaviour of the sample. This allows for better and faster equipment testing and sample processing. Especially for harsh environments, e.g. during launch of a sounding rocket or parabolic flights, as well as for autonomous operation under microgravity conditions, PSDs should be replaced by a camera system bundled with image processing software.

Commercially available smart-cameras including imageprocessing hardware could be adapted to perform sample de- 
tection. The focus on the system presented here is the flexibility to easily customize it for different detection scenarios. Additionally, the system needs to fit in a rocket payload with restrictions in size and weight. The complete ESL facility on MAPHEUS has a length of $1200 \mathrm{~mm}$, a diameter of $390 \mathrm{~mm}$, and a mass of $95 \mathrm{~kg}^{13}$. The use of individual small cameras in combination with image processing on the existing controlcomputer gives great benefit.

Hence, the current setup of the newly developed microgravity electrostatic levitator utilises two Basler acA640-120uc cameras connected via USB3.0 to a mini-ITX computer. Within the current setup $260 \mathrm{px} \times 260 \mathrm{px}$ at $200 \mathrm{fps}$ are used to represent a field of $7 \mathrm{~mm} \times 7 \mathrm{~mm}$. We aim for using higherframe-rate cameras in the future. This enhancement will allow to investigate smaller samples with lower mass, which will accelerate faster in the electrical field.

To make use of the acquired images for positioning control, a real-time image analysis is necessary to determine the sample position. Using a $200 \mathrm{fps}$ acquisition hardware, the algorithm needs to perform several steps to derive the sample position from the camera image within $5 \mathrm{~ms}$.

First the image needs to be acquired from the camera buffer. This process depends mainly on the camera linkage and the device driver. The current setup allows for a bandwidth of up to $300 \mathrm{MB} / \mathrm{s}$ per channel.

Depending on the contrast in the image, the detection should recognise if the sample is present. This step is especially needed in autonomous environments to automatically load the next sample and start a new measurement cycle.

For lower temperature range a background illumination is needed to offer sufficient contrast for the sample detection algorithm. For higher temperatures the illumination needs to be switched off to see the bright sample on a dark background. These cases need to be identified by the algorithm.

In the next step the algorithm calculates the sample position. This part needs to be robust with respect to sample size, position and shape. For example it needs to find fast moving samples, which no longer appear as a spherical object with clear border (motion blur) to offer a chance for the controller to react properly.

\section{ALGORITHM}

There are multiple algorithms available to detect circular contours in images. The most common ones are based on the Hough Transformation ${ }^{21}$. To find a circle in a greyscale picture, an edge detection needs to be performed first. Therefore, the first order image derivatives are calculated. It is done by applying an operator (for comparison of different operators see Ref. 22) to each pixel of an image. The two image derivatives need to be combined to one greyscale image then highlighting the edges of the source image. For each detected edge pixel it is determined if it fulfils the circle equation. If it is fulfilled, the element in the parameter space $x, y, r$ is voted. The highest voted elements are likely to define a circle in the image $^{21}$.
This algorithm can easily be used to find all circles with different radii in one single image. The efficiency of the Hough Transformation depends mainly on the edge detection algorithm and the predefined parameter space. After the replacement of the PSDs by CCDs first tests with commercial detection algorithms based on Hough Transformation were performed. The results show a good detection rate when a precise edge of the sample can be found. The detection fails for images containing motion blur. Further tests showed, the provided algorithms lack of performance when used for feedback control. Especially, if there are multiple objects in the acquired image, the algorithm tries to perform a circle detection for each. This approach results in varying detection rates and it cannot be guaranteed that the positions are send in real-time to the feedback controller. The detection of a single levitating sample is a better-defined task. At any given time there is only one sample which needs to be identified. Furthermore, this sample is the solitary object within the detection range and has an elliptical shape. Paying attention to those preconditions, a much more specific sample detection algorithm was developed.

Fig. 1 shows one example for the detection process for an unheated sample with a diameter of $1.6 \mathrm{~mm}$ in front of an illuminated background. In Fig. 1a the image acquired from the camera is shown. The detection range has a size of $240 \mathrm{px} \times 240 \mathrm{px}$; so there remains a border of $10 \mathrm{px}$ at each side of the image.

In a first step the algorithm needs to test if background illumination is turned on. Therefore, the average grey value of the left border area $(240 \mathrm{px} \times 10 \mathrm{px})$ is calculated. If the grey value is lower than the threshold of 127 , the algorithm assumes a bright sample in front of a dark background and the image gets inverted for further tasks. In the given example the average value is higher than the threshold, so the image remains unmodified for further processing. In parallel, to identify the detection mode, the border is cropped from the image. Then the remaining detection area is divided into squares of $15 \mathrm{px} \times 15 \mathrm{px}$. For each of the squares the sum of all contained pixel values is computed resulting in a $16 \times 16$ matrix. Fig. 1b shows those sums normalised to 8 bit greyscale. Since there is considered only one major object, the darkest square represents a part of the sample. This step ensures that smaller objects in the detection region like the foil in the lower left corner or inhomogeneity in the illumination are excluded from the sample detection. Furthermore, the difference between the minimum and maximum in the intensity matrix is used to identify whether a sample is present at all or is considered lost from levitation by checking against a predefined minimum contrast.

The centre of the identified square marks a random pixel within the borders of the sample. It represents an estimation of the sample position in the image. Consequently, the algorithm starts from this position to search for the border of the sample in four directions. The search range is two times the defined maximum sample radius (see Fig. 1c). The border detection is now performed by an edge detection on pixel-basis. Therefore, the first derivative in four directions is calculated. The highest gradient in each of the four directions defines one 

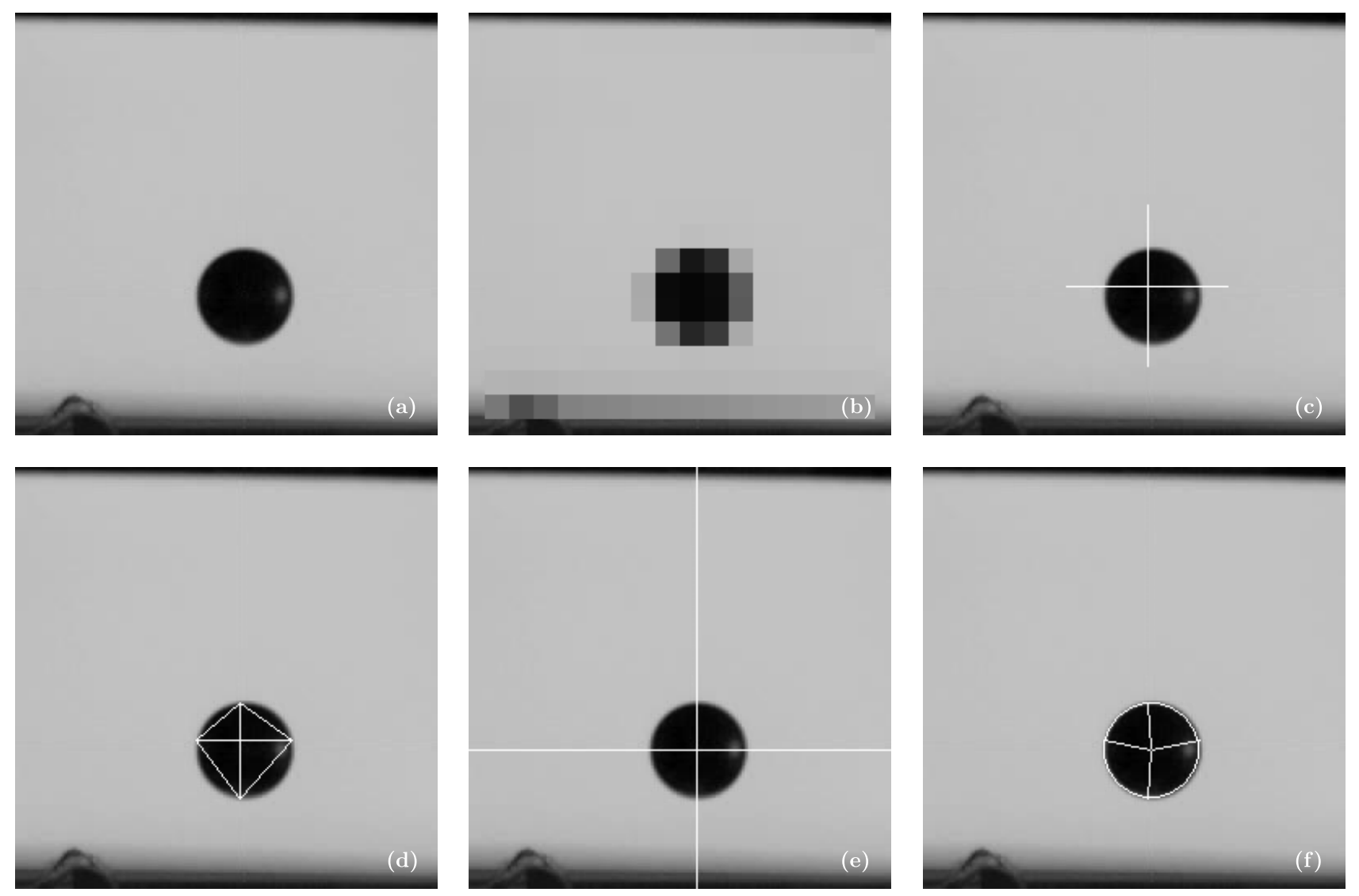

FIG. 1. Different steps performed by the sample detection software to find an unheated sample $(\varnothing \approx 1.6 \mathrm{~mm})$ in front of an illuminated background. (a) Image acquired from camera system, (b) Grey-value sums of the $15 \mathrm{px} \times 15 \mathrm{px}$ regions scaled to 8 -Bit, (c) Range to detect the border of the sample based on the lowest sum of grey values, (d) Detected edges identified by highest grey-value gradients in each direction, (e) Calculated centre of levitating sample, (f) Estimated sample-shape based on the calculated radius

pixel at the edge of the sample. The detected points from the example are highlighted in Fig. 1d.

From these four points the coordinates of the samples centre is calculated by averaging horizontal and vertical coordinates, respectively (Fig. 1e). Within the current setup, the centre can be detected with a spatial resolution of $27 \mu \mathrm{m}$ in each direction. The uncertainty results from the border detection and can be estimated to $\pm 1.5 \mathrm{px}$ which corresponds to $\pm 40 \mu \mathrm{m}$.

Finally, this algorithm is applied to each of the two cameras. This results in the three dimensional sample position, which is represented by the three coordinates of the sample centre. These values are transmitted to the feedback controller to stabilise the sample position. The sample loss information is forwarded to the sequence control and will trigger feeding in the next sample.

In addition the sample radius can be estimated by calculating the average distance between the sample centre and the detected border points. At present it is used to check the value against a predefined limit to proof the identified object probably is the sample. The estimated sample-shape based on the calculated radius is shown in Fig. If and confirms the detected sample centre.

The detection might be influenced by the relation between sample position and camera focus, irregular shape and rota- tion of a solid sample, and by light reflections on the surface. All these disturbances can be neglected, for an incandescent, liquid sample close to the centre of the electrode system.

For the system used here, the algorithm needs to complete in $5 \mathrm{~ms}$ for both cameras. In addition to the steps described above, the image sequences should be stored on a hard disk drive for later analysis and be displayed live on the user screen. The described algorithm is implemented in NI LabVIEW 2017 using the IMAQdx libraries. The calculated sample position is encoded and sent via a RS232 interface to the feedback controller.

The algorithm is able to perform more than 4000 cycles/s using one core of an Intel i7 processor. It offers enough scope for future utilisation of faster camera systems and thereby faster control algorithms and better observation of sample.

\section{CONTROL MODEL}

To implement a suitable controller for stabilising the sample position it is necessary to find a control model for the electrostatic levitator. For laboratory systems using five controllable and one grounded electrodes a model is described in Refs. 1 and 19. The ELF facility also uses six electrodes 


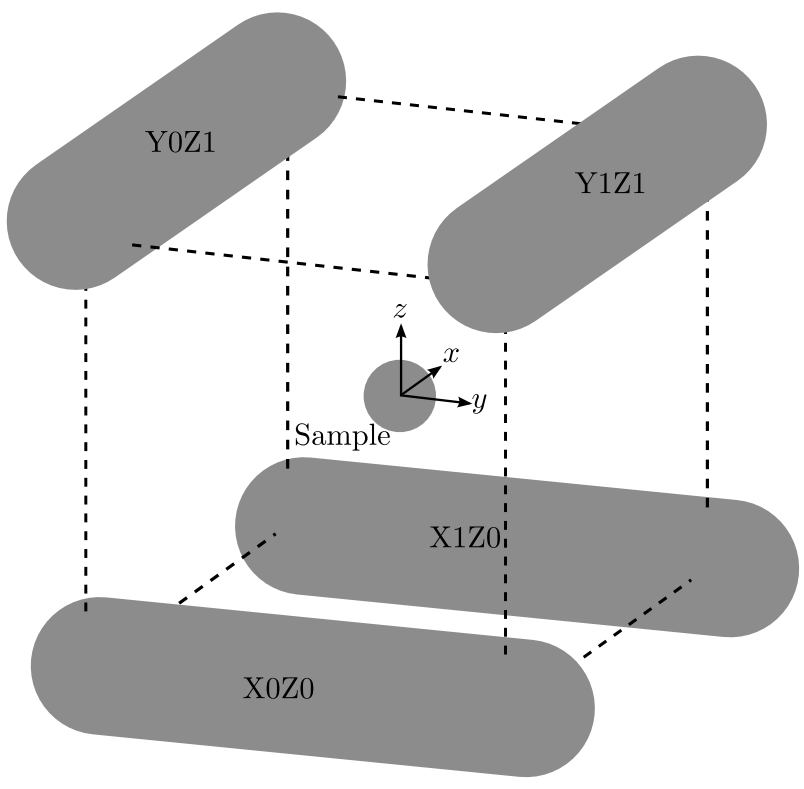

FIG. 2. Electrode system of the microgravity electrostatic levitator with four separate electrodes to control the three-dimensional position of the levitated sample ${ }^{24}$. In the current setup, the distance between the centres of the electrodes is $10 \mathrm{~mm}$ and the electrodes have a diameter of $3 \mathrm{~mm}$.

arranged orthogonally ${ }^{17,23}$. In all those cases the control system can be separated into three one-dimensional problems. In Ref. 14 a tetrahedral electrode-system using four controllers is presented. This setup requires a conversion between the Cartesian camera-coordinates and the electrode positions. All mentioned configurations cannot offer accessibility from all sides to the levitation area.

A new electrode system ${ }^{24}$, using only four electrodes was developed to meet the environmental conditions of microgravity. The setup allows the direct usage of the Cartesian cameracoordinates for the control system and offers full accessibility to the levitating sample from all six planes. In order to gain stable control of the levitating sample inside this electrode system, a new control model needs to be formulated.

The outputs of the system model are the three spatial coordinates $x, y, z$ in $\mathrm{mm}$ of the sample position. The inputs are the voltages of the four electrodes $U_{X 0 Z 0}, U_{X 1 Z 0}, U_{Y 0 Z 1}, U_{Y 1 Z 1}$ in V. Fig. 2 shows the geometric conditions in the electrode system model with a sample in the origin.

The sample is considered as a point with an electrical charge of $q_{P}$ in As and a mass $m$ in $\mathrm{kg}$. If it is exposed to the electrical field $\vec{E}$ of a point electrode, motion results from electrostatic, inertial, gravitational, and frictional forces.

Under microgravity conditions and using a vacuum chamber, the gravitational and frictional forces can be neglected. The acceleration $a$ of the sample can be calculated by Eq. (1).

$$
\vec{a}=\frac{q_{P}}{m} \vec{E}
$$

For the controller model, the electrodes are considered as point charges with position in the middle of each rod electrode. The electric field of each electrode can be calculated by
Coulomb's law. For the resulting acceleration of the sample, the fields of all electrodes can be calculated as the superposition of the four fields. In addition, the calculation of the electrical field needs to consider the image charges on each electrode. For the rod electrodes, the positions of those charges are inside the electrodes. For a sample position in the origin the superposition of the electrical fields of the image charges neutralises.

The resulting non-linear differential equation, describing the movement of the charged sample in the electrostatic field, is given in Eq. (2). $Q_{E_{i}}$ is the charge of the electrode, $\varepsilon_{R}$ the relative permittivity, $\varepsilon_{0}$ the vacuum permittivity and $\vec{r}$ the position vector in the field.

$$
\vec{a}=\overrightarrow{\ddot{r}}=\frac{q_{P}}{4 \pi \varepsilon_{0} \varepsilon_{R} m} \sum_{i=1}^{4} \frac{Q_{E_{i}} \vec{r}_{i}}{\left\|r_{i}\right\|^{3}}
$$

Eq. (2) uses the charges of the electrodes as model input. Using high-voltage amplifiers, it is only possible to control the potential of the electrodes but not the charges. Both parameters correlate linearly.

The controller is needed to stabilise the sample position in the centre position between the electrodes. It means the setpoint for the desired position is constant. Considering Eq. (2) and a possible steady gravitational force, the electric charges of the electrodes need to be constant to achieve an unstable equilibrium. A combination of sample position $x_{0}, y_{0}, z_{0}$ and the voltages on the electrodes $U_{0, X 0 Z 0}, U_{0, X 1 Z 0}, U_{0, Y 0 Z 1}, U_{0, Y 1 Z 1}$ is defined as the operating point of the system. In ideal circumstances it is identical to the unstable equilibrium. For controller design, the nonlinear model should be linearised around this point using first order Taylor series.

The resulting linear model for the sample movement by the electrostatic field is given in Eq. (3). In these equations the positions $\Delta x, \Delta y, \Delta z$ and the voltages $\Delta u_{X 0 Z 0}, \Delta u_{X 1 Z 0}, \Delta u_{Y 0 Z 1}, \Delta u_{Y 1 Z 1}$ describe the deviations from the predefined operating point. The directions $x, y$ and $z$ as well as the indices of the potentials $u$ refer to Fig. 2. $P$ and $K$ are linear factors resulting from constants in Eq. (2) and from the linearisation.

$$
\begin{aligned}
\Delta \ddot{x}-P_{x} \Delta x= & K_{x}\left(\Delta u_{X 0 Z 0}-\Delta u_{X 1 Z 0}\right) \\
\Delta \ddot{y}-P_{y} \Delta y= & K_{y}\left(\Delta u_{Y 0 Z 1}-\Delta u_{Y 1 Z 1}\right) \\
\Delta \ddot{z}-P_{z} \Delta z= & K_{z}\left(\left(\Delta u_{X 0 Z 0}+\Delta u_{X 1 Z 0}\right)\right. \\
& \left.-\left(\Delta u_{Y 0 Z 1}+\Delta u_{Y 1 Z 1}\right)\right)
\end{aligned}
$$

The linearisation shows good approximation in the range of $\pm 2 \mathrm{~mm}$ around the operating point along each axis. Considering the radius of the used samples from $0.6 \mathrm{~mm}$ to $1.1 \mathrm{~mm}$ it is enough to calculate a controller for stabilising the sample position.

\section{CONTROLLER}

The model developed in the previous section is the basis for the controller implementation. In Ref. 19 a gain scheduling 
controller for electrostatic levitator on ground is presented. The controller uses an estimation of the sample charge to adapt the control parameters. This estimation needs a constant gravitational force and is not applicable for microgravity conditions. In addition, the existing electrostatic levitation facilities described in Refs. 15 and 19 use five or six controllable electrodes and for each axis the electrodes are separated. In the specific microgravity setup ${ }^{24}$, only four electrodes are present. The developed controller needs to share electrodes to control the sample position in the three axes as shown in Eq. (3).

According to the unstable, linear model presented in Eq. (3), a PID controller for each axis is used for the microgravity electrostatic levitator.

The differential part is necessary to obtain a stable closed loop feedback control. The levitating sample needs to be in the middle of the electrode system as shown in Fig. 2 to allow proper heating and temperature measurement. Therefore, an integral action in the controller is required.

With Eq. (3) and the standard PID-controller equation, the closed control loop can be calculated. Studies with a swinging sample were used to adjust the unknown parameters of the linearised model. In combination with the closed loop equation, the controller parameters can be calculated to gain stability and robustness for the sample positioning. The outputs of the three PID controllers are three differential voltages $\Delta u_{x}, \Delta u_{y}$ and $\Delta u_{z}$. To calculate the output of each highvoltage-amplifier, they have to be portioned among the four electrodes and the operating point needs to be added. The corresponding equations are presented in Eq. 4.

$$
\begin{aligned}
& U_{X 0 Z 0}=U_{0, X 0 Z 0}-0.5 \Delta u_{x}-0.25 \Delta u_{z} \\
& U_{X 1 Z 0}=U_{0, X 1 Z 0}+0.5 \Delta u_{x}-0.25 \Delta u_{z} \\
& U_{Y 0 Z 1}=U_{0, Y 0 Z 1}-0.5 \Delta u_{y}+0.25 \Delta u_{z} \\
& U_{Y 1 Z 1}=U_{0, Y 1 Z 1}+0.5 \Delta u_{y}+0.25 \Delta u_{z}
\end{aligned}
$$

The control algorithm is implemented on a National Instruments CompactRIO system with a Virtex-5 LX 110 FPGA (Field Programmable Gate Array) running at $500 \mathrm{~Hz}$. The control software is written in NI LabVIEW using the FPGA Toolbox. The sample position is received by the FPGA from the detection computer as a four byte array via serial interface. In this array, three bytes represent the sample position in the detection range of $240 \mathrm{px}$ for three axes. The fourth byte indicates the frame end and includes information about status of background illumination and possibly lost samples. If no sample is detected, the controller uses the positions from the previous iteration.

To gain the most current position, the last four bytes in the buffer are analysed. The controller runs faster than the sample detection cameras. If the camera was not able to send a new sample position, the controller continues with the previous one. The received positions in pixels are converted to deviations from the centre of the electrode system in $\mathrm{mm}$.

The FPGA calculates the potential differences for each of the three axes using the time discrete version of the PIDcontroller. Then outputs for the four amplifiers are calculated. In the next step, the voltage is limited to the respective maxi- mum output of the amplifiers. The last step is the calculation of the control voltage by dividing the limited output by the gain factor.

The analogue voltage output is realised using the high speed National Instruments C-series module 9263 with an accuracy of 16 bit. In addition, the measured positions and the calculated output are transferred to the host system for data logging using FPGA FIFOs. After receiving a predefined number of consecutive information on a lost sample, the host system is starting to load and process the next one.

\section{PERFORMANCE TESTS}

For microgravity experiments, all electrodes are operating in the same voltage range of $\pm 3 \mathrm{kV}$. For ground experiments, the two amplifiers connected to the bottom electrodes are replaced to offer up to $20 \mathrm{kV}$ for lifting the sample. Additionally, the sample release method differs between microgravity and laboratory. In microgravity, the sample is hold between two rods and released by pulling both rods apart (see Fig. 3). It takes up to 3 frames for the rods to move out of the sample detection area. During this time, the rods may disturb the sample detection and the position controller cannot be used. Assuming a free fall, in those $15 \mathrm{~ms}$, the sample would move more than $1 \mathrm{~mm}$, which is one third of the levitation area. The controller directly needs to apply a high voltage on the lower electrodes to catch the falling sample. If the sample is not sufficiently charged it will get lost. Tests showed, that levitation starting with a sample placed on the lower rod (like done in Ref. 25) are much more reliable on ground. In microgravity, the initial sample movement depends only on small residual accelerations resulting from the vehicle or the movement of the rods. Under this condition, there is enough time for the controller to capture it.

Multiple tests were performed on ground to obtain the desired levitation stability. The experiments showed that the charge of the sample is not reproducible. It depends on sample material, surface, contact between sample and rod and other parameters which are not directly accessible. So the parameter $q_{P}$, used to build the model in Eq. (2), is unknown. In laboratory environment an in-loop estimation of the sample charge based on the potential difference along the gravitational axis could be used ${ }^{19}$. This method is not possible in microgravity. High speed camera recordings of the levitation process on ground show that the sample bounces multiple times on the electrode before it finally lifts off. If the sample is not charged sufficiently, it will fall back onto the electrode and recharge.

After successful positioning tests in laboratory, the system was tested under microgravity conditions at the ZARM drop tower in Bremen. Due to the different sample release method (Fig. 3), multiple contacts of the sample with the electrode cannot be performed. As a result, the initial charge of the released sample varies between the experiments. In experiments at the drop tower, it could be observed that even applying the maximum field strength, some samples were not influenced by the electrostatic field.

For samples with sufficient charge, the sample detection 


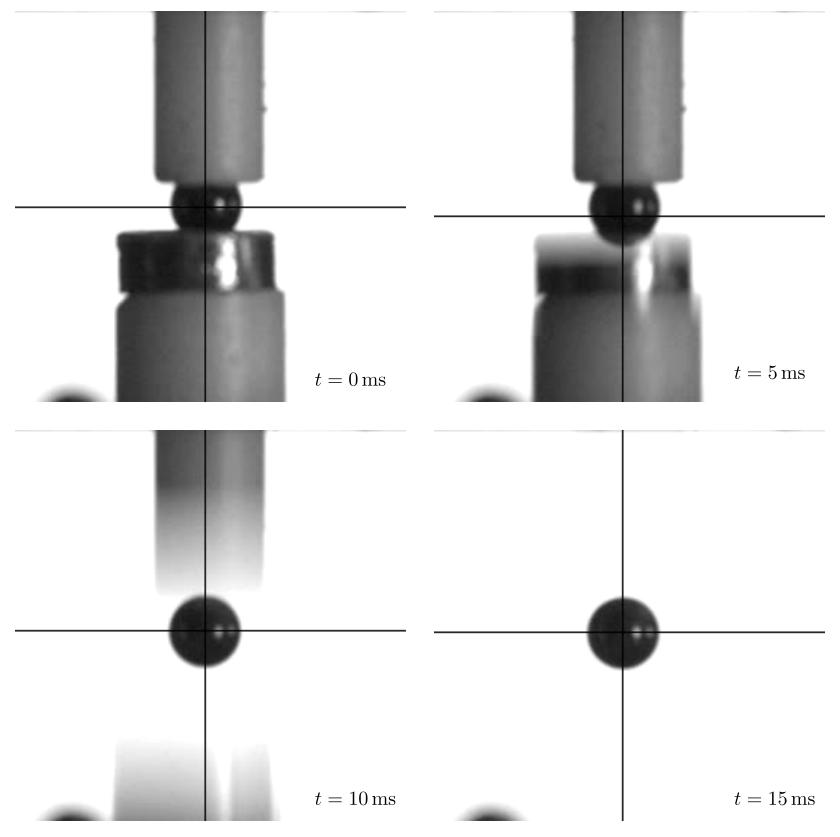

FIG. 3. Sample release mechanism in microgravity performed during a parabolic flight

algorithm and controller showed good performance. Fig. 4 shows a successful levitation process of a $\mathrm{Zr}_{64} \mathrm{Ni}_{36}$ sample with a diameter of $1.9 \mathrm{~mm}$ and a mass of $25 \mathrm{mg}$ in a drop tower. The three coordinates of the sample and the associated electrode potentials are depicted. For microgravity conditions, the operating point was set to $1500 \mathrm{~V}$ for all electrodes and the output was limited to the range from $0 \mathrm{kV}$ to $3 \mathrm{kV}$. The sample was released at $0 \mathrm{~s}$ and at $4.1 \mathrm{~s}$, the capsule with the experiment reached the deceleration chamber and the microgravity phase ended. The sample was not heated because of the short drop time of $4.74 \mathrm{~s}$.

During the release, an initial speed in direction of $+x$ was measured on the sample. The recovery time is about $1.5 \mathrm{~s}$. After $3 \mathrm{~s}$, only small, unavoidable position changes due to the system instability remain. The overall control stability was $\pm 50 \mu \mathrm{m}$ for all axes. It includes a minor detection error resulting from the rotation of the not completely spherical sample. The voltage spikes in Fig. 4 result from the differential part of the PID-controller. Due to the sample detection, the position can only change in discrete steps of $27 \mu \mathrm{m}$. With the controller parameters used during the experiment, a voltage change of approx. $600 \mathrm{~V}$ is calculated and portioned among the electrodes, as given in Eq. 4.

Even under small residual accelerations (in the order of $\left.10^{-6} \mathrm{~g}\right)^{26}$, the full field strength of $3 \mathrm{kV}$ was reached during the levitation.

Further developments focused on obtaining a higher initial surface charge. Better results were achieved by improving the contact between the sample and the rod and by preheating. Additionally the limitations of the electrode potentials were removed to offer a field strength of $6 \mathrm{kV}$ and the delay between sample release and controller reaction was minimized. Those improvements were successfully tested on the $28^{\text {th }}$ DLR parabolic flight campaign. Compared to the drop tower, residual accelerations are much higher (approx. $\left.10^{-2} \mathrm{~g}\right)^{27}$. During the campaign, levitation was performed on preheated $\mathrm{Zr}_{64} \mathrm{Ni}_{36}(\varnothing \approx 1.4 \mathrm{~mm}, m \approx 10 \mathrm{mg})$ and $\mathrm{La}_{80} \mathrm{Cu}_{20}$ $(\varnothing \approx 1.4 \mathrm{~mm}, m \approx 9 \mathrm{mg})$ samples.

In 2017, the experiment was onboard the MAPHEUS 6 sounding rocket. The sample detection worked as expected but due to a failure of one high voltage amplifier, offering only limited voltage output, only a few samples could be levitated successfully.

\section{CONCLUSION AND OUTLOOK}

This paper describes a fast and reliable way to measure and control the sample position for a microgravity electrostatic levitator. Conventionally used PSD-based sampledetection systems turned out to be unreliable in harsh environments. Hence, for microgravity electrostatic levitation, a CCD-camera-based system is used and proved to offer more reliable results.

In order to determine the $3 \mathrm{~d}$ sample position from the acquired images, a sample detection algorithm was developed. It is designed to identify fast moving single spherical objects. While other less specialised algorithms do not meet the performance requirements, the presented one is by now approximately twenty times faster than the current image acquisition hardware. It offers the possibility to use camera systems with higher frame rates or resolution in future developments.

Three PID controllers implemented on a FPGA are used to stabilise the three dimensional sample position within the electrode system. The calculated control model in combination with pretesting was successfully used to obtain parameters for a stable feedback control system. The facility was successfully tested in laboratory environment and under microgravity conditions in the drop tower, on parabolic flights and on MAPHEUS sounding rocket.

${ }^{1}$ W. K. Rhim, S. K. Chung, D. Barber, K. F. Man, G. Gutt, A. Rulison, and R. E. Spjut, Rev. Sci. Instrum. 64, 2961 (1993).

${ }^{2}$ W.-K. Rhim, K. Ohsaka, P.-F. Paradis, and R. E. Spjut, Review of Scientific Instruments 70, 2796 (1999).

${ }^{3}$ Y. S. Sung, H. Takeya, and K. Togano, Journal of Applied Physics 92, 6531 (2002).

${ }^{4}$ T. Ishikawa, P.-F. Paradis, T. Itami, and S. Yoda, Measurement Science and Technology 16, 443 (2005).

${ }^{5}$ S. Mukherjee, W. L. Johnson, and W. K. Rhim, Applied Physics Letters 86, 014104 (2005).

${ }^{6}$ R. W. Hyers and J. R. Rogers, High Temperature Materials and Processes 27 (2008), 10.1515/htmp.2008.27.6.461

${ }^{7}$ J. Brillo, A. I. Pommrich, and A. Meyer, Physical Review Letters 107 (2011), 10.1103/physrevlett.107.165902.

${ }^{8}$ T. Kordel, D. Holland-Moritz, F. Yang, J. Peters, T. Unruh, T. Hansen, and A. Meyer, Physical Review B 83 (2011), 10.1103/physrevb.83.104205.

${ }^{9}$ N. A. Mauro, M. Blodgett, M. L. Johnson, A. J. Vogt, and K. F. Kelton, Nature Communications 5 (2014), 10.1038/ncomms5616.

${ }^{10} \mathrm{P}$. Heintzmann, F. Yang, S. Schneider, G. Lohöfer, and A. Meyer, Applied Physics Letters 108, 241908 (2016).

${ }^{11}$ S. Jeon, D.-H. Kang, Y. H. Lee, S. Lee, and G. W. Lee, The Journal of Chemical Physics 145, 174504 (2016).

${ }^{12}$ S. Earnshaw, Trans. Camb. Phil. Soc. 7, 97 (1842).

${ }^{13} \mathrm{C}$. Neumann, Entwicklung eines elektrostatischen Levitators für den Einsatz in Mikrogravitation, Ph.D. thesis, Universität des Saarlandes (2017). 

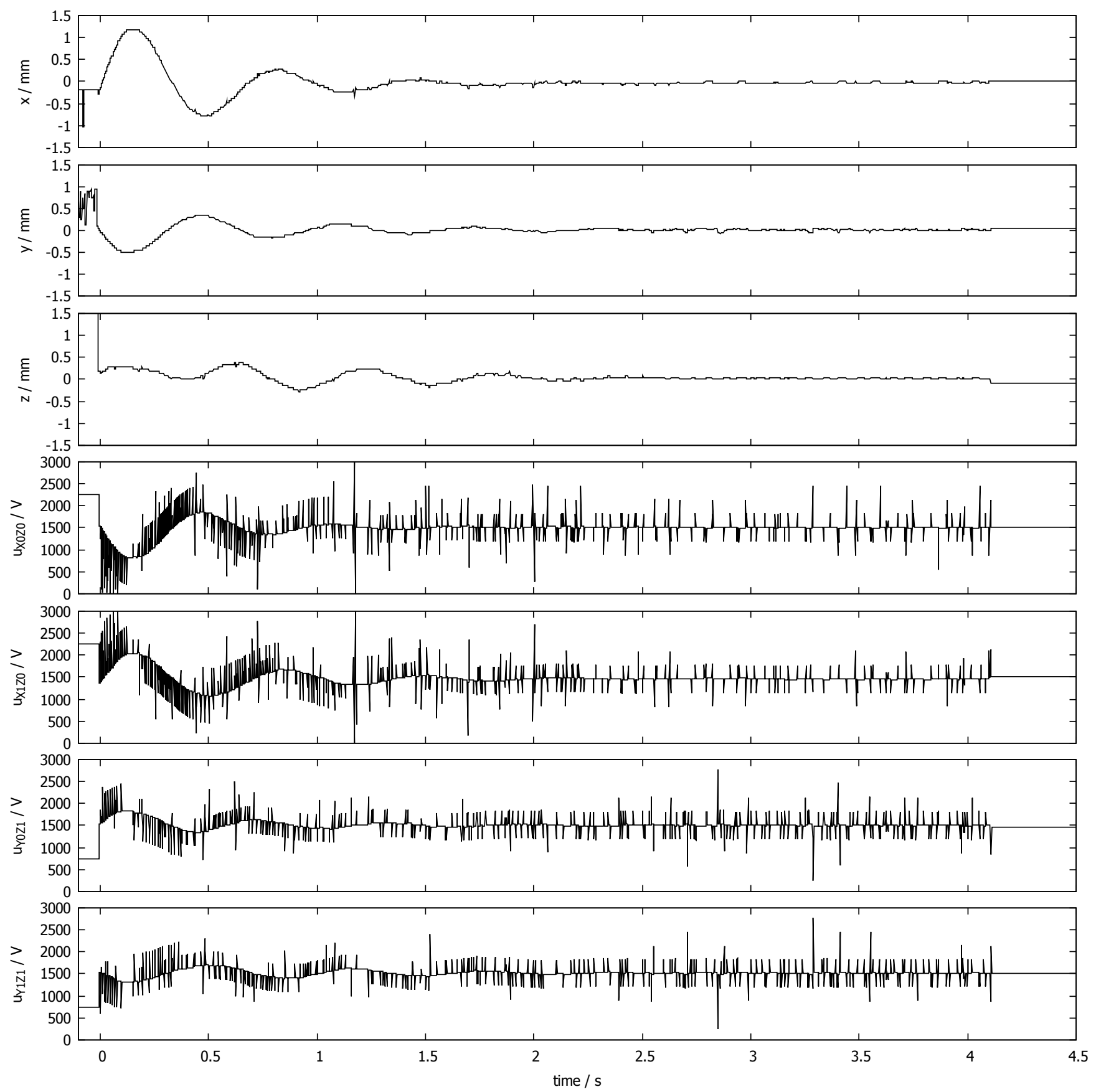

FIG. 4. Microgravity positioning-test using a $\mathrm{Zr}_{64} \mathrm{Ni}_{36}$ sample $(\varnothing \approx 1.9 \mathrm{~mm}, m \approx 25 \mathrm{mg})$ at the ZARM drop tower in Bremen. Sample is released at $t=0 \mathrm{~s}$ and the capsule is decelerated starting at $t \approx 4.1 \mathrm{~s}$. The upper three diagrams show the detected position of the sample and the lower four diagrams show the resulting electrode potentials.

${ }^{14}$ W. K. Rhim, M. Collender, M. T. Hyson, W. T. Simms, and D. D. Elleman, Review of Scientific Instruments 56, 307 (1985).

${ }^{15}$ Y. Nakamura, K. Murakami, K. Shibasaki, H. Tamaru, H. Ohkuma, S. Yukizono, T. Ishikawa, J. Okada, T. Takada, Y. Sakai, T. Arai, and N. Fujino, in Proc. 64th International Austronautical Congress, IAC-13,A2,7,8,x16553 (2013).

${ }^{16}$ T. Ishikawa, J. T. Okada, P.-F. Paradis, and V. K. Marahalli, Transactions Of The Japan Society For Aeronautical And Space Sciences, Aerospace Technology Japan 12, Th_15 (2014).

${ }^{17}$ H. Tamaru, C. Koyama, H. Saruwatari, Y. Nakamura, T. Ishikawa, and T. Takada, Microgravity Science and Technology 30, 643 (2018).
${ }^{18}$ A. J. Makynen, J. T. Kostamovaara, and R. A. Myllyla, in Optics, Illumination, and Image Sensing for Machine Vision IV, edited by D. J. Svetkoff (SPIE, 1990).

${ }^{19}$ T. Meister, H. Werner, G. Lohöfer, D. Herlach, and H. Unbehauen, Control Engineering Practice 11, 117 (2003).

${ }^{20}$ R. R. Hale, ed., Optical Sample-Position Sensing For Electrostatic Levitation, Vol. 1118 (1989).

${ }^{21}$ R. O. Duda and P. E. Hart, Communications of the ACM 15, 11 (1972).

${ }^{22}$ R. Maini and H. Aggarwal, International Journal of Image Processing 3, 1 (2009).

${ }^{23}$ T. Nakamura, Transactions of the Japan Society for aeronautical and space 
sciences, space technology Japan 5, 17 (2007)

${ }^{24}$ C. Neumann, "Elektrostatischer Levitator sowie Messsystem mit einem elektrostatischen Levitator," Patent (2014).

${ }^{25}$ T. Meister, Aufbau und Regelung eines elektrostatischen Levitators, Ph.D. thesis, Ruhr-Universität Bochum (2000).
${ }^{26}$ H. Dittus, Endeavour 15, 72 (1991).

${ }^{27}$ V. Pletser, S. Rouquette, U. Friedrich, J.-F. Clervoy, T. Gharib, F. Gai, and C. Mora, in 66th International Astronautical Congress 2015 (IAC 2015): Space - The Gateway for Mankind's Future (2015). 
(a)

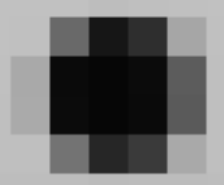

$\theta$ (b)

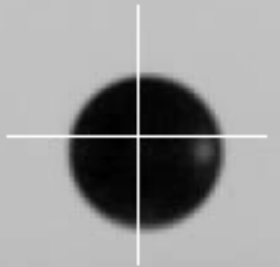

(c)

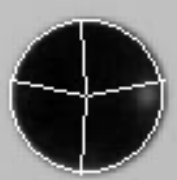

(d)

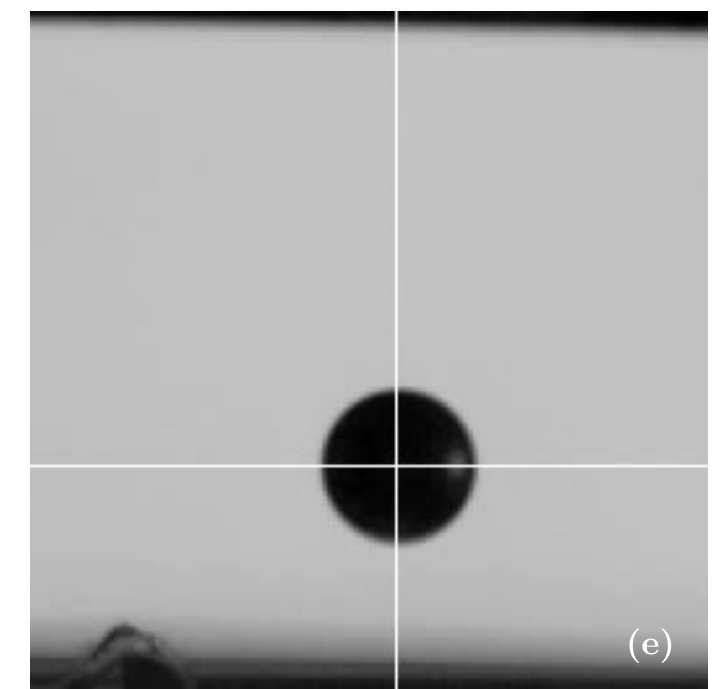

(f) 


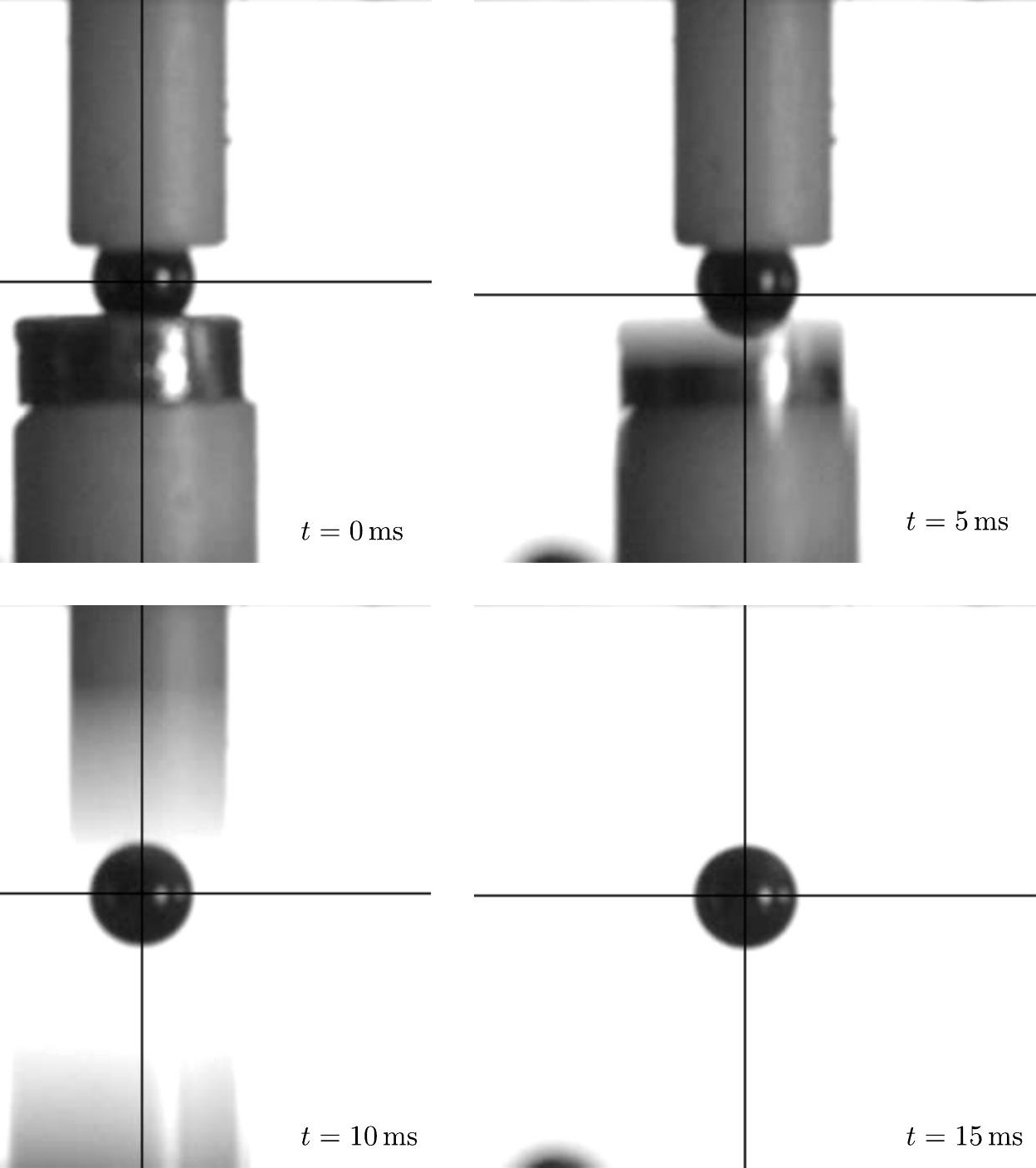


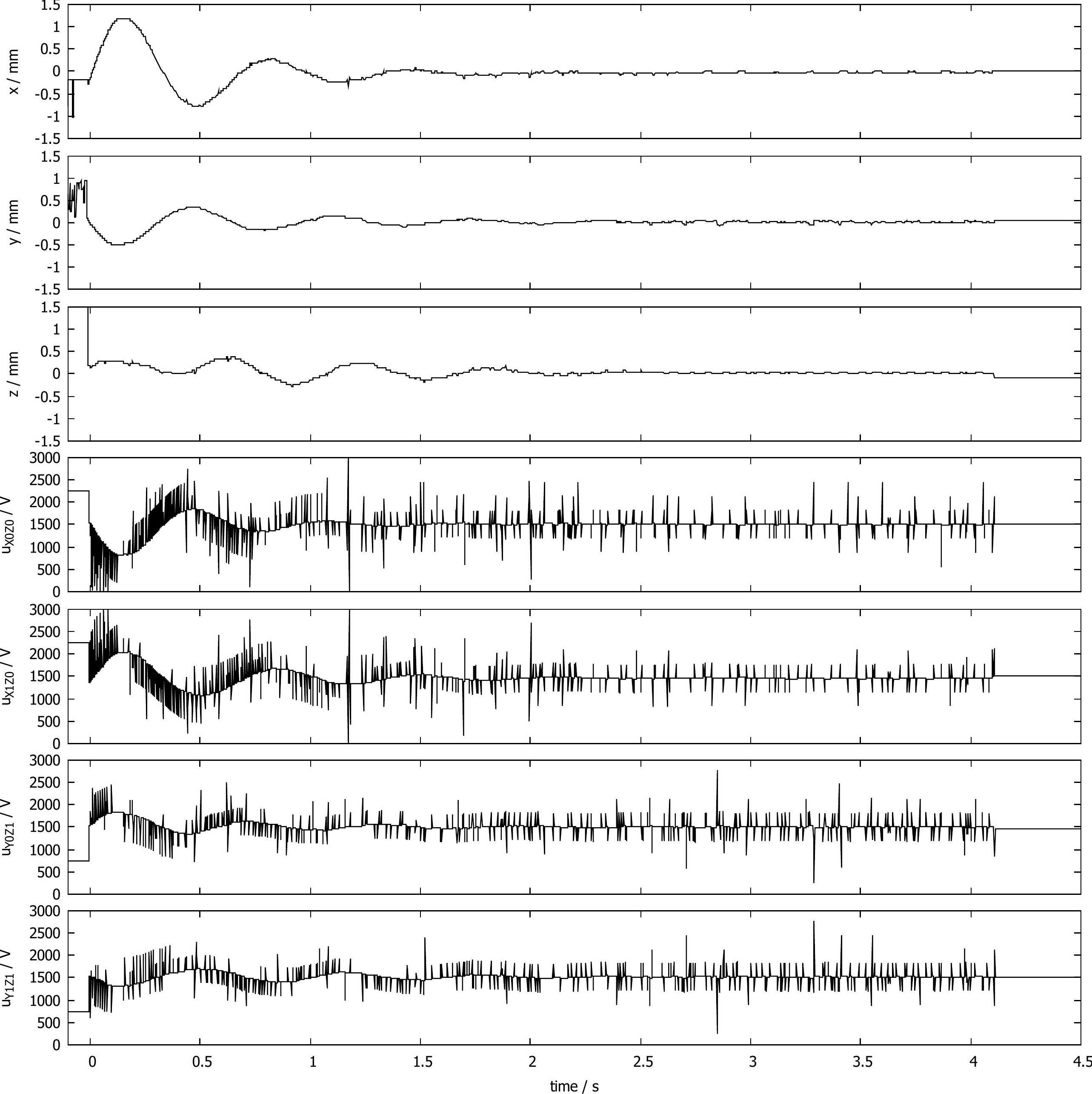

